\title{
Order in Nanometer-Thick Intergranular Films at Au-Sapphire Interfaces
}

\author{
M. Baram, *** S.H. Garofalini, ${ }^{* * *}$ W.D. Kaplan, ${ }^{*}$ \\ * Department of Materials Engineering, Technion - Israel Institute of Technology, Haifa, \\ 32000, Israel \\ ** School of Engineering and Applied Sciences, Harvard University, Cambridge, MA, 02138 \\ *** Department of Materials Science and Engineering, Rutgers University, Piscataway, NJ, \\ 08854-8065
}

The existence of intergranular film (IGFs) at ceramic grain boundaries was observed over 30 years ago. IGFs were found to have a strong influence on material properties (e.g. enhancing fracture toughness or degrading high temperature strength). Recently it was proposed that interfaces can be treated in a manner similar to bulk phases and can have a distinct structure and composition at equilibrium (termed as "complexions") [1]. IGFs were identified as one type of complexion. It was also found that the films' properties can change depending on structure and thickness of the film. However, due to microscopy limitations the structure of the film was not fully resolved and considered to be mainly disordered (amorphous), although order was predicted in the films from both theoretical models and simulations [2]. Relatively recently IGFs were discovered to also exist at metal-ceramic interfaces, and the fundamental questions regarding the structure of the film remain the same. In addition, the reason for the stability of the film at equilibrium is still not fully clear. While it is the common opinion that IGFs reduce interfacial energy, this was never experimentally proven in a direct manner.

A model experiment was used to form equilibrated $\mathrm{Au}$ particles in contact with sapphire, in the vicinity of small droplets of anorthite glass $\left(\mathrm{CaO}-2 \mathrm{SiO}_{2}-\mathrm{Al}_{2} \mathrm{O}_{3}\right)$ [3]. This method provided specimens from which the interface energy could be measured, and correlated with the local atomistic structure and chemistry. The local structure was characterized using aberration corrected transmission electron microscopy (TEM) from site-specific cross-section specimens prepared by a modified technique in a dual-beam focused ion beam system [4]. Quantitative aberration corrected HRTEM analysis was conducted where negative spherical aberration (Cs) and exit wave reconstruction were correlated with simulations to determine the atoms' location in the bulk, and the order in the film. One example of such an analysis is presented in Fig. 1. In addition, the equilibrium thickness of the films was measured from the amplitude and phase of the reconstructed wave function. It was found that since the amplitude and phase holds information on the chemistry and the crystallography respectively, their combination provides an accurate method to measure the IGF thickness.

Order was indeed observed in the $1.2 \mathrm{~nm}$ thick films adjacent to the sapphire crystal. From comparison to simulations [2] the order was concluded to be in the form of "Ca cages", experimentally demonstrating that ordering is an intrinsic part of IGFs, as predicted from molecular dynamics and diffuse-interface theory. This finding together with the measurement of the equilibrium thickness and interface energy offers additional experimental support to the perception of IGFs as complexions and their future incorporation in bulk phase diagrams. 


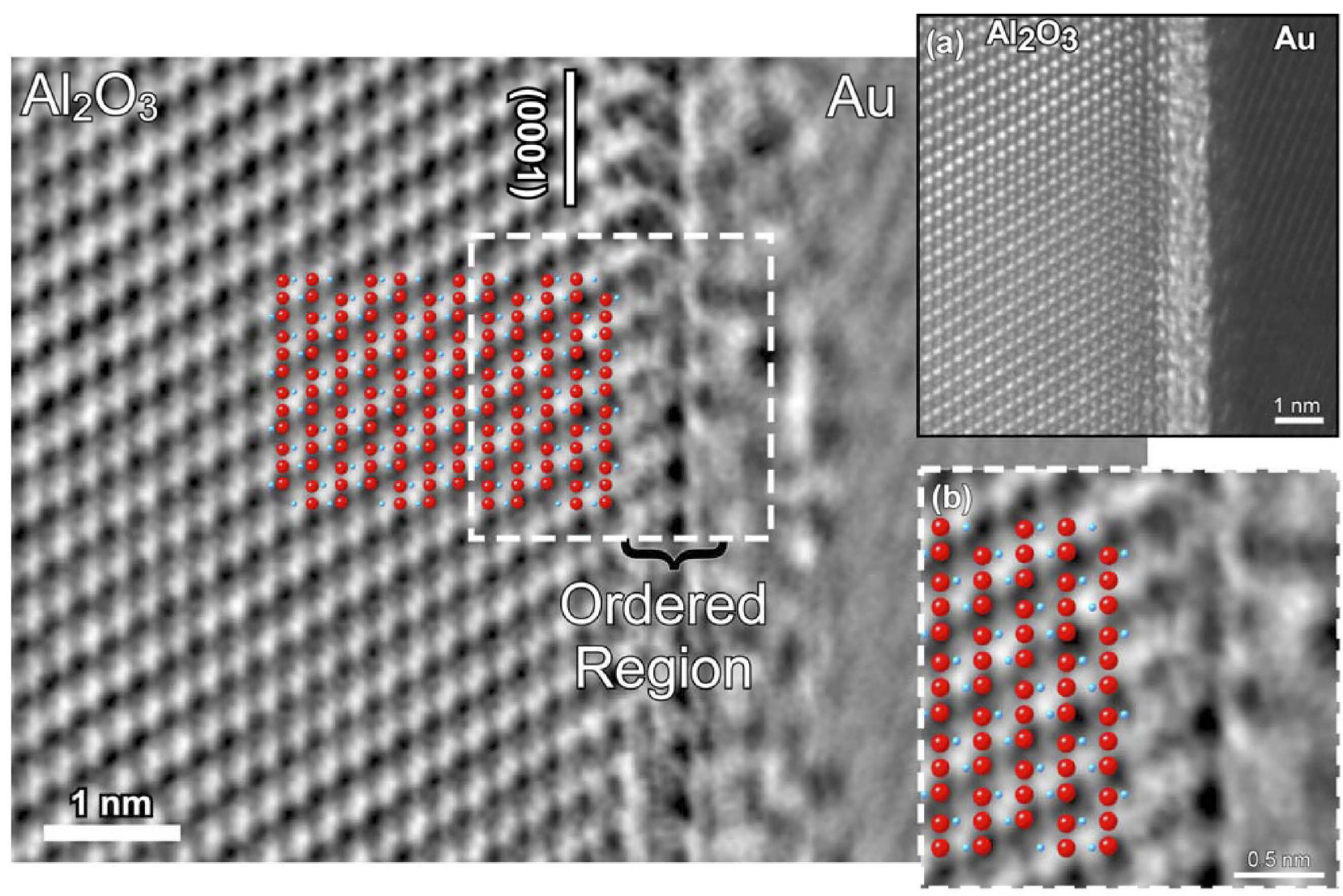

FIG. 1. Reconstructed phase from an experimental defocus series $(\mathrm{Cs} \sim 1 \mu \mathrm{m})$. a. single HRTEM micrograph and $b$. magnification of the region indicated with a white dashed line. The ordered region in the film can clearly be detected. In addition, fitting of atom locations was conducted from comparison to simulations where $\bullet-$ oxygen and $\bullet-$ aluminum. Sapphire zone axis: [11 $\overline{2} 0]$.

\section{References}

[1] M. Tang et al., Phys. Rev. B, 73(2) (2006) 1

[2] S. Zhang et al., J. Phys. Chem. B., 110(5) (2006) 2233

[3] M. Baram et al., J Mater. Sci., 41(23) (2006) 7775

[4] M. Baram et al., J Microsc. (Oxf), 232(3) (2008) 395

[5] The authors would like to thank Y. Kauffmann for fruitful discussions. This work was supported by the Israel Science Foundation (\#163/05) and the Russell Barrie Nanotechnology Institute at the Technion. MB acknowledges support from the Israeli Ministry of Science via an Eshkol Fellowship, and SHG acknowledges support from DOE BES, Division of Materials Sciences grant DE-FG02-O6ER46336. 